\title{
Corrigendum
}

\section{Quantification of galantamine in Narcissus tazetta and Galanthus nivalis (Amaryllidaceae) populations growing wild in Iran- CORRIGENDUM}

Majid Rahimi Khonakdari, Mohammad Hossein Mirjalili, Abbas Gholipour, Hassan Rezadoost and Mahdi Moridi Farimani

doi:10.1017/S1479262117000107. Published online by Cambridge University Press 14 March 2017.

The address for Abbas Gholipour was incorrectly published as:

Department of Biology, Payam Noor University (PNU), Sari, Mazandaran, Iran

The correct address for Abbas Gholipour is:

Department of Biology, Payame Noor University, Tehran, Iran.

\section{Reference}

Majid Rahimi Khonakdari, Mohammad Hossein Mirjalili, Abbas Gholipour, Hassan Rezadoost and Mahdi Moridi Farimani (2017) Quantification of galantamine in Narcissus tazzeta and Galanthus nivalis (Amaryllidaceae) populations growing wild in Iran. Published online by Cambridge University Press 14 March 2017. Doi: 10.1017/S1479262117000107. 